\title{
Charged particle identification in the ALICE experiment
}

\section{Eugenio Nappi ${ }^{*}$}

INFN, Sezione di Bari

Via G. Amendola, 173, Italy

E-mail: eugenio.nappi@ba.infn.it

\begin{abstract}
A key feature of the ALICE experiment will be the capability to identify the charged particles in the full range of momentum thus allowing to investigate the properties of the quark-gluon plasma during its dynamical evolution toward the freeze-out phase when the re-hadronization occurs. Low momentum hadron identification will be achieved via measurements of energy loss in the silicon layers of the inner tracking system and in the gas of the largest volume TPC ever built so far. At higher momenta, identification will be performed by employing the TOF barrel based on multigap resistive plate chambers with an intrinsic time resolution of about 60 ps and an array of RICH detectors equipped with large area CsI photocathodes. Transition radiation detectors consisting of xenon-filled time expansion chambers with a radiator stack of carbon fibers will identify electrons with momentum above $1 \mathrm{GeV} / \mathrm{c}$, near mid-rapidity, to study the suppression of quarkonia decaying in electron pairs and the production of heavy quarks (charm, beauty) in their semileptonic decays. Characteristics and expected performance of each particle identification system will be reviewed.
\end{abstract}

European Physical Society

HEP2005 International Europhysics Conference on High Energy Physics EPS (July $21^{\text {st }}-27^{\text {th }} 2005$ ) in Lisboa, Portugal

\footnotetext{
${ }^{*}$ Speaker (on behalf of the ALICE Collaboration)
} 


\section{Introduction}

Head-on collisions between ultrarelativistic heavy nuclei are believed to provide the extreme conditions of energy densities sufficient for the transition of hadronic matter to a shortlived state, called Quark-Gluon Plasma (QGP), where quarks are no longer confined within the nucleon. The forthcoming Large Hadron Collider, LHC, scheduled to start commissioning during 2007, will be the ultimate facility for searching the QGP.

Complementary to the CMS and ATLAS experiments whose physics programmes focus on the search of the Higgs particle and super-symmetric particles in p-p collisions, ALICE (A Large Ion Collider Experiment) has been designed to study nucleus-nucleus collisions at LHC energies, in conditions significantly more favourable than those achieved at SPS and RHIC, via a simultaneous measurement of many different observables [1].

The main feature of the ALICE layout is the combined capability to track and identify charged particles in a wide momentum range. The anticipated very high multiplicity of the final states in central $\mathrm{Pb}-\mathrm{Pb}$ collisions, implying an occupancy that locally could rise to such a level as to spoil the particle identification system's performance, presented a serious challenge in the detector design. Moreover the goal to achieve a particle separation power greater than $3 \sigma$ on a track-by-track basis, necessary to keep the contamination below a 10\% level, required the development of devices based on advanced technologies, which nowadays represent the stateof-art in their respective application fields.

\section{The ALICE layout}

The ALICE setup, shown in Fig.1, consists of a central $(|\eta| \leq 0.9)$ complex of tracking and PID detectors and a muon spectrometer in the forward rapidity region $(2.4 \leq \eta \leq 4)$. The following devices, starting from the beam axis, are sheathed in the inherited L3 solenoid magnet:

- an inner tracking system (ITS) with six cylindrical layers of highly accurate positionsensitive silicon detectors designed to track charged particles emerging from the main interaction vertex and to identify decay products of short-lived secondary particles having strange and charm quark content;

- a cylindrical time projection chamber (TPC) envisaged to determine the charged particle trajectories curving in the magnetic field, allowing particle momentum and charge to be measured. With its volume of $88 \mathrm{~m}^{3}$, it is the largest gas detector ever built so far;

- two highly segmented particle identification barrel arrays. The inner array consists of 540 transition radiation detector (TRD) modules arranged in six layers of xenon-filled time expansion chambers with a radiator stack of carbon fibers optimized to provide the best compromise between TR yield, radiation thickness and mechanical stability. The second array of 1638 double-stack multigap resistive plate chambers (MRPC) with an intrinsic time resolution of about $60 \mathrm{ps}$ is devoted to the hadron identification via TOF measurements;

- a single arm array of seven Ring Imaging CHerenkov detectors (RICH) exploiting the novel technology of manufacturing large area CsI photocathodes performs an inclusive 
identification of high momentum charged hadrons (High Momentum Particle Identification Detector, HMPID);

- a single arm outer electromagnetic calorimeter (PHOton Spectrometer, PHOS) located below the central barrel region.

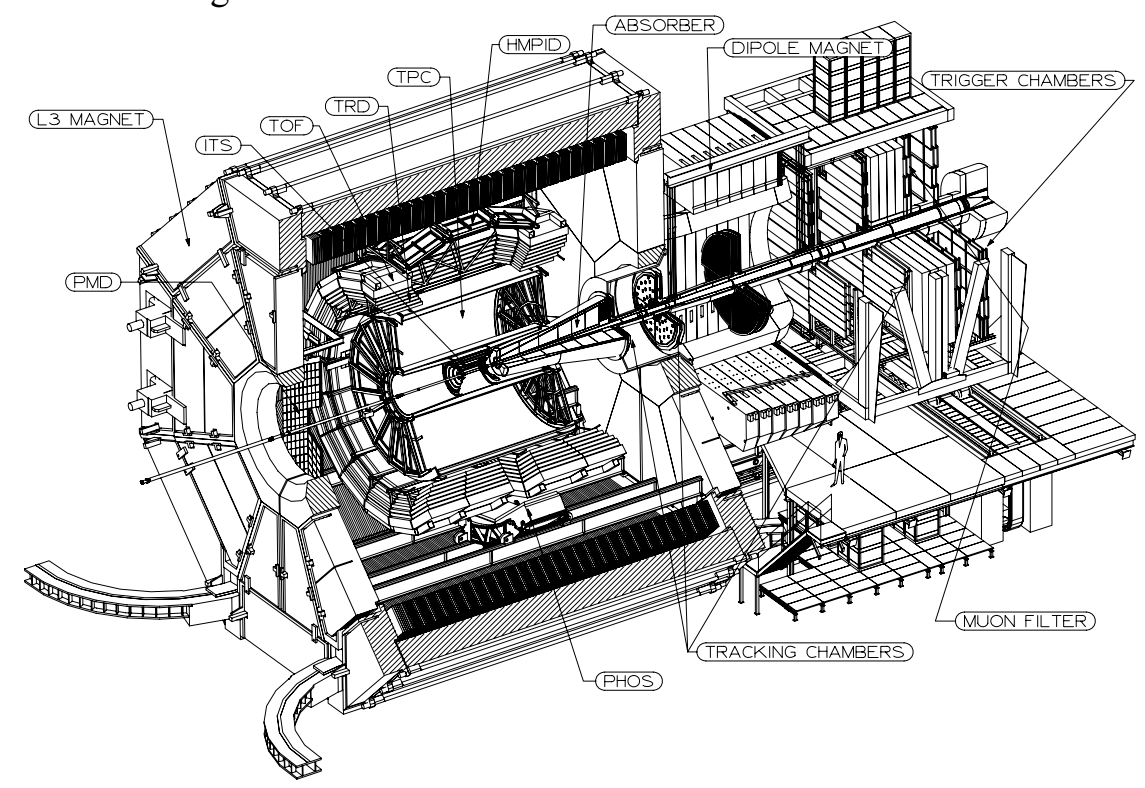

Fig. 1. Axonometric view of the ALICE layout.

The forward muon arm, featuring measurements of quarkonia states with a $\Upsilon$ mass resolution of about $100 \mathrm{MeV} / \mathrm{c}^{2}$, consists of a complex arrangement of absorbers (reaching totally about $10 \lambda_{\text {int }}$ ), a large dipole magnet ( $3 \mathrm{Tm}$ integral field), ten stations of thin multi-wire proportional chambers (MWPC) equipped with highly segmented cathode planes for tracking and four Resistive Plate Chambers (RPCs) for muon identification and triggering.

Four small and very dense zero degree calorimeters, made of tungsten and lead with embedded quartz fibres read out by photomultipliers, are located about $100 \mathrm{~m}$ downstream in the machine tunnels on both sides of the interaction region to measure and trigger upon the impact parameter. Photon multiplicity is measured by the Photon Multiplicity Detector (PMD). Additional detectors, designed to provide fast trigger signals and event multiplicity at large rapidity, complete the ALICE set-up.

\section{The performance of PID detectors}

In $\mathrm{Pb}-\mathrm{Pb}$ central collisions, $97 \%$ of the many thousands of particles produced per event will have a $p_{t}<2 \mathrm{GeV} / \mathrm{c}$, the bulk of the remaining $3 \%$ will be in the range $2-5 \mathrm{GeV} / \mathrm{c}$ although a not negligible fraction $(\sim 0.02 \%)$ will have a $\mathrm{p}_{\mathrm{t}}>5 \mathrm{GeV} / \mathrm{c}$. Therefore more than one detector will be employed in ALICE to perform an efficient and unambiguous particle identification, as shown in Fig. 2. Low momentum hadron identification is achieved via measurements of energy loss in the four silicon layers equipped with an analogue readout (two drift and two microstrip silicon detector layers) and in the TPC gas, featuring a $\mathrm{dE} / \mathrm{dx}$ resolution of $10 \%$ and $6.8 \%$ respectively. Above the $1 / \beta^{2}$ region, identification is performed by employing the TOF barrel 
(overall time resolution $<90 \mathrm{ps}$ ). The HMPID, optimized to achieve a $\sigma_{\beta} / \beta=10^{-3}$, will extend the useful range for the identification of $\pi / \mathrm{K}$ and $\mathrm{K} / \mathrm{p}$ up to $3 \mathrm{GeV} / \mathrm{c}$ and $5 \mathrm{GeV} / \mathrm{c}$ respectively. Finally, the TRD will supplement the ITS and TPC electron/pion identification by a pion rejection factor of the order of 100 at momenta in excess of $1 \mathrm{GeV} / \mathrm{c}$.

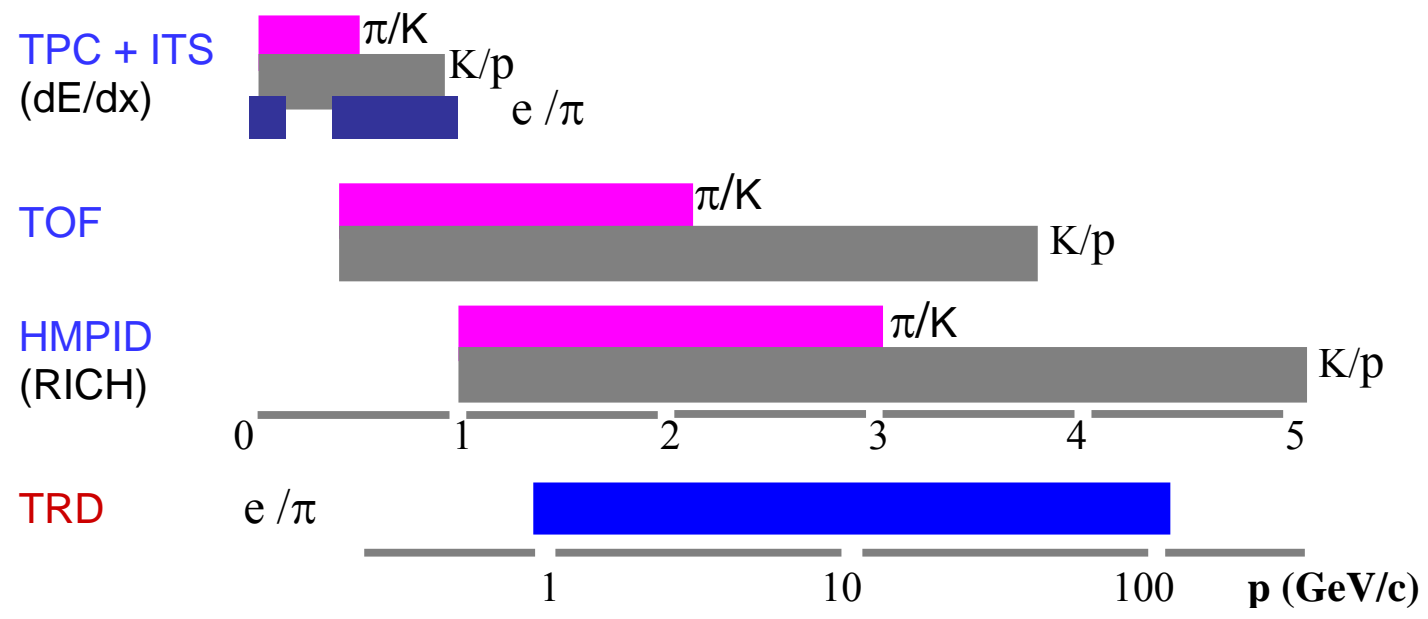

Fig. 2. Momentum intervals in which the various ALICE PID detectors allow to achieve a $3 \sigma$ separation level.

In the momentum regions where more PID detectors are simultaneously able to provide a response, a Bayesian approach to combine the various signals is currently under study [2]. Results obtained so far are quite promising as shown in Fig. 3.

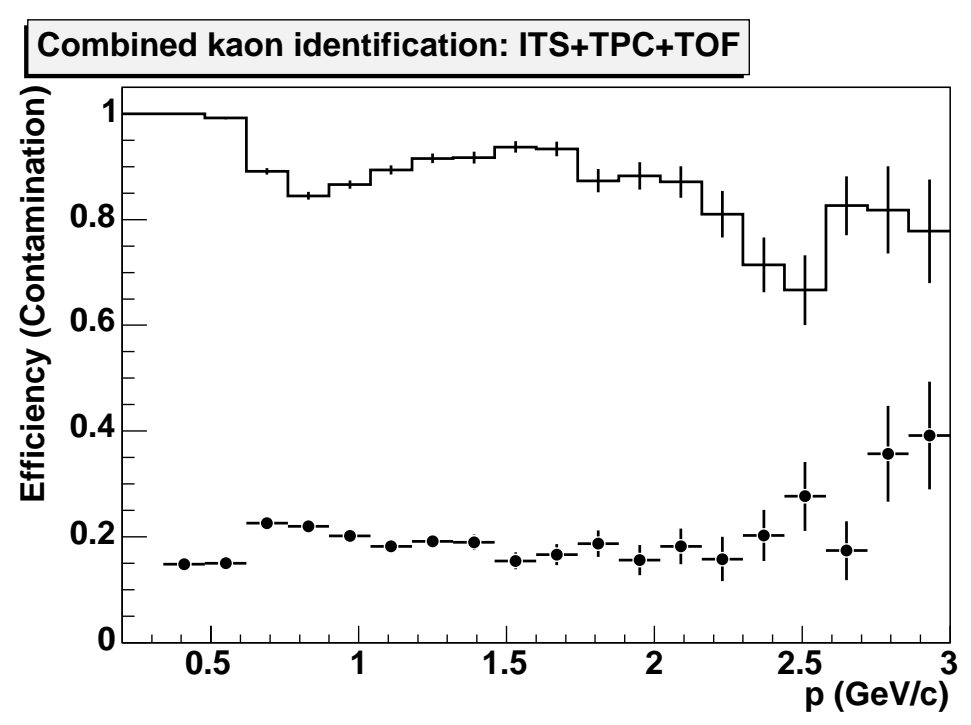

Fig. 3. Efficiency and contamination for kaon identification as obtained by combining together three PID detectors.

\section{References}

[1] ALICE Collaboration, Physics performance report, Volume I CERN/LHCC 2003-049

[2] ALICE Collaboration, Physics performance report, Volume II CERN/LHCC 2005-049 